\title{
Active vibration control of a thin rectangular plate in air or in contact with water in presence of tonal primary disturbance
}

\author{
S. Carra ${ }^{\mathrm{a}, *}$, M. Amabili ${ }^{\mathrm{a}}$, R. Ohayon ${ }^{\mathrm{b}}$, P.M. Hutin ${ }^{\mathrm{c}}$ \\ ${ }^{a}$ Industrial Engineering Department, University of Parma, Via G.P. Usberti 181/A, 43100 Parma, Italy \\ b Structural Mechanics and Coupled Systems Laboratory, Conservatoire National des Arts et Métiers, \\ 2 rue Conté, 75003 Paris, France \\ c Office National d'Études et de Recherches Aérospatiales (ONERA)/DDSS, BP 72 - 29 avenue de la Division Leclerc, \\ 92322 Chatillon cedex, France \\ Available online 17 October 2007
}

\begin{abstract}
A rectangular aluminium plate vibrating in air or in contact with water is investigated. The plate is bolted to a wall of a rectangular Plexiglas container. Some modal analyses are firstly realised in order to verify the effects of different water levels and of the control components on the modal parameters. A filtered-x least mean square (FXLMS) adaptive feedforward algorithm is then applied to the system, realising structural vibration control in linear field with a SISO approach on the first vibration modes of the plate in presence of tonal primary disturbance.
\end{abstract}

Keywords: Active vibration control; Tonal disturbance; Fluid-structure interaction; Filtered-x LMS; Piezoelectric actuators

\section{Introduction}

In the present study, active vibration control of a thin-walled rectangular aluminium plate, representing a flexible wall of a thick Plexiglas container, is experimentally investigated in linear field for both empty and water-filled tank, using five piezoelectric Lead-Zirconate-Titanate (PZT) patches as control actuators. Some preliminary experimental modal analyses are also performed on the plate for different levels of fluid in the tank.

The use of active control techniques for the suppression of vibrations of very light structures is in fact a very important target in many applications, where the additional masses of stiffeners or dampers should be avoided. Active techniques are also more suitable in cases where the disturbance to be cancelled or the properties of the controlled system vary with time. Moreover, aeronautical and space structures often present thin walls containing fuel, where the fluid-structure interaction must be taken into account. The presence of water increases the modal mass of the system and free surface waves complicate

\footnotetext{
* Corresponding author. Tel.: +390521 906029; fax: +390521905705.

E-mail address: silvia.carra@libero.it (S. Carra).
}

the system dynamics, as reported by Amabili [2]. Morand and Ohayon [12] also deeply explain the effects of fluid-structure interactions on the dynamics of structure.

In the last years, several studies have been dedicated to the case of control of fluid-loaded structures. Some of them are specifically dedicated to the active control of sound radiation. Other studies also investigate the application of active control on structural vibrations of fluid-loaded structures. Lee and Park [10] used a near-field approach to simulate the active control of sound radiation from a fluid-loaded rectangular plate, obtaining high global reductions both in radiated pressure and plate response. Li and Zhao [11] numerically studied the active control of both structural vibration and acoustic radiation of a fluid-loaded laminated plate with active damping control and active constrained layer damping (ACLD). Active control of a fluid-loaded plate has been also recently investigated by Fein et al. [6], who have proposed a modal control technique, which has been experimentally applied on a plate in order to reduce vibrations on its first natural frequencies.

In this paper, the Least Mean Square adaptive feedforward algorithm, as introduced by Widrow and Stearns [15], is applied to the system to be controlled in the form of filtered-x LMS (FXLMS) control. This modified version of the classical LMS 


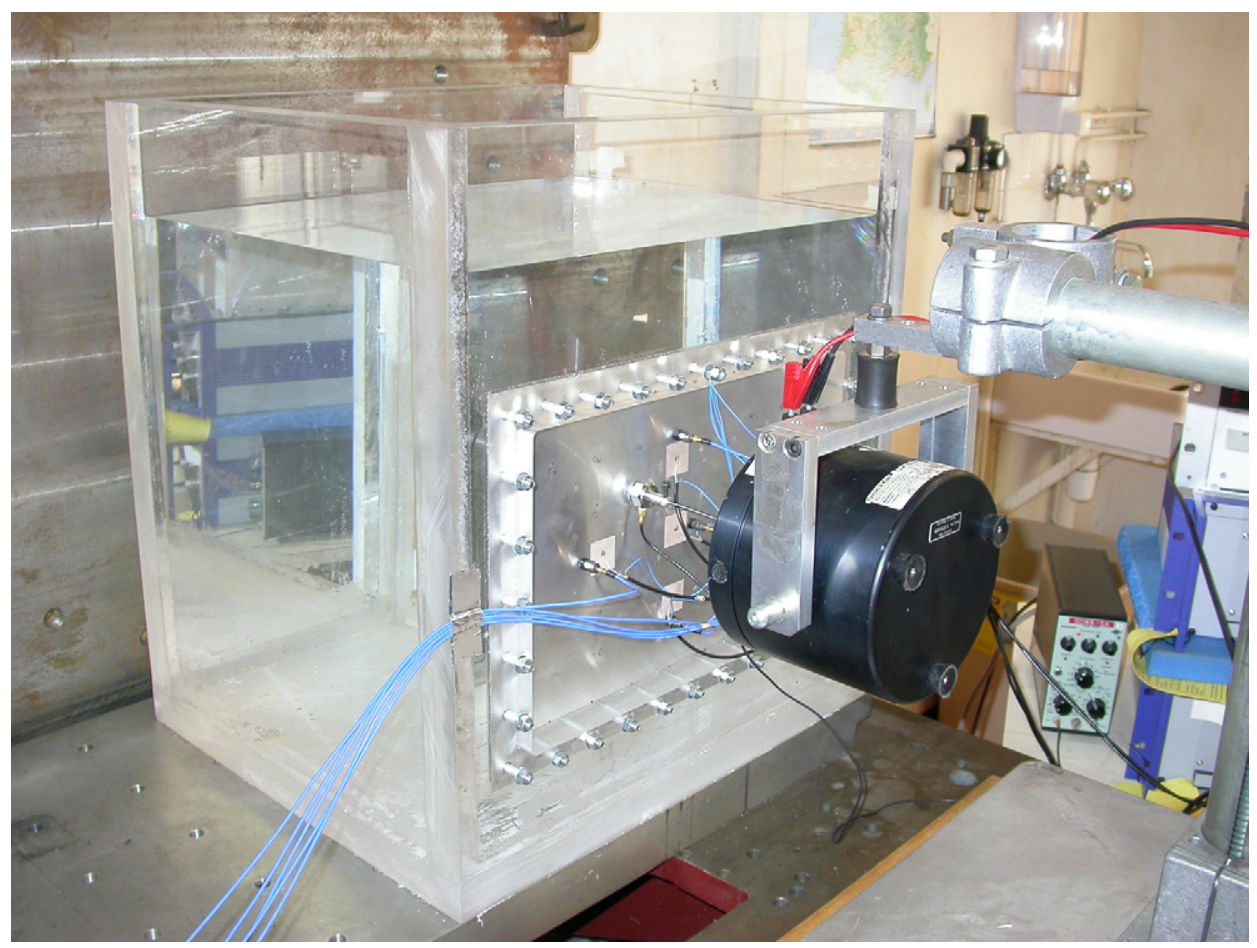

Fig. 1. The system and the experimental set-up for active vibration control (case of water-filled tank).

algorithm is in fact widely used in active control systems, since it allows to compensate for the presence of a secondary path transfer function after the adaptive filter [9].

In this study, the use of FXLMS control is limited to the case of tonal primary disturbance. The secondary control input is generated by some piezoelectric PZT actuators; Dimitriadis et al. [4] firstly showed the effectiveness of piezoelectric actuators for vibration suppression on two-dimensional structures.

The positioning of the PZT patches is here based on the knowledge of the natural modes of the plate and, particularly, of their deformation energy. The error sensors (accelerometers) are positioned very closed to the piezoelectric patches, in order to obtain a nearly-collocated system. The optimisation of the position of actuators and sensors is in fact of fundamental significance for the success of the control [5]. Peng et al. [13] proposed a performance criterion for the optimisation of the piezoelectric patch actuators locations on a thin cantilever plate, showing the consequent positive effects on FXLMS control effectiveness by numerical simulations. Petitjean et al. [14] experimentally compared the control effectiveness of FXLMS feedforward technique and collocated feedback control strategies for active structural acoustic control using PZT actuators on a sandwich honeycomb panel. They showed that, for single frequency primary excitation, the optimisation of the location of actuators and error sensors can produce a significant increase of the control effectiveness.

The aim of the present research is therefore to experimentally test active feedforward control on a nearly-clamped plate using piezoelectric actuators, both in presence and in absence of fluid-structure interaction, in order to reduce structural vibrations due to tonal disturbance on the first resonances.

\section{The system}

The system to be controlled is constituted by a thin-walled rectangular aluminium plate $(400 \mathrm{~mm} \times 283 \mathrm{~mm} \times 0.8 \mathrm{~mm})$, which is bolted to a wall of a Plexiglas container in an opening with dimensions $350 \mathrm{~mm} \times 233 \mathrm{~mm}$, at a distance of $70 \mathrm{~mm}$ from the internal bottom of the tank (Fig. 1). The long edges of the plate are parallel to the tank bottom.

The boundary conditions are assumed to be as near as possible to those of clamped edges. A stainless-steel frame, made by four different parts with width $20 \mathrm{~mm}$ and thickness $10 \mathrm{~mm}$, placed on the edges of the opening on the inside of the Plexiglas wall, is used in order to increase the stiffness of the structure on the plate boundaries, which can be therefore considered quite rigid in the low-frequency range. Silicone has been also inserted, in order to guarantee watertight. The stainless-steel frame, the aluminium plate and the Plexiglas wall are connected by thirty-two stainless-steel flathead screws placed on through holes and fixed by nuts on the outside of the Plexiglas wall. The number of screws has been chosen in order to make the boundary conditions of the plate as uniform as possible and in order to avoid the escape of liquid.

The internal dimensions of the tank are $440 \mathrm{~mm} \times 350 \mathrm{~mm} \times$ $500 \mathrm{~mm}$. The five Plexiglas walls are quite thick $(30 \mathrm{~mm})$ and they are connected by a particular glue (specific for Plexiglas) that is designed to withstand water at room temperature. The Plexiglas container also presents a good transparency in order to allow a better monitoring of the inner plate surface.

A tap has been also placed on the bottom of the back wall of the tank, in order to easily empty the container after each test in presence of water. 


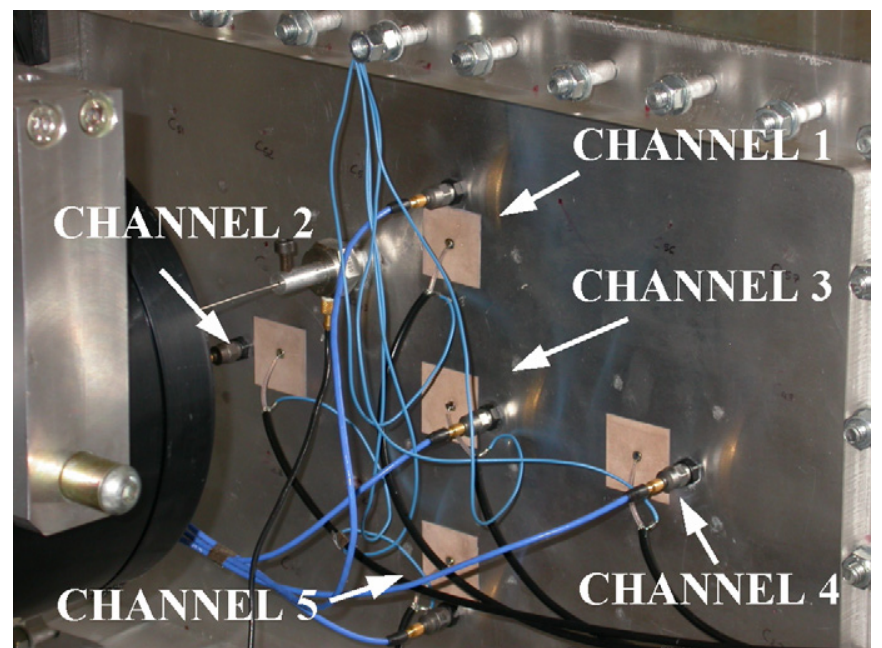

Fig. 2. The five control channels.

\section{The control components}

Before applying control, the necessary control components (sensors and actuators) are glued on the plate surface. They are constituted by five piezoelectric Lead-Zirconate-Titanate (PZT) patches $(25 \mathrm{~mm} \times 25 \mathrm{~mm} \times 0.2 \mathrm{~mm}$, PIC 255 material, with electrical capacity equal to $50 \mathrm{nF}$, from Physik Instrumente) and five PCB accelerometers (model M352C66, weight $2 \mathrm{~g}$, with sensitivity equal to about $10 \mathrm{mV} / \mathrm{ms}^{-2}$ ).

Every accelerometer is positioned close to a PZT actuator, realising a nearly-collocated control system. Every couple accelerometer-piezoactuator represents therefore a channel of the control system and it is numbered as shown in Fig. 2. PZT patches and accelerometers represent respectively the secondary control actuators and the error sensors of the control system; they are placed on the same side of the plate surface (air side).

The adopted PZT actuators are subject to a contraction of $180 \mu \varepsilon$ at $200 \mathrm{~V}$, which has been verified to be enough in order to realise control in linear field (that is for low vibration amplitude) on the studied structure. Every actuator receives the command signal from a high voltage power amplifier (model E-507.00, from Physik Instrumente).

A load cell (model B\&K 8200, weight $21 \mathrm{~g}$, with sensitivity $3.81 \mathrm{pC} / \mathrm{N}$ ) is also glued on the plate surface in the excitation point, where the primary disturbance is applied during all the control tests and during the experimental modal analyses. Its presence is fundamental in order to monitor the actual force that is applied to the plate.

Every PZT patch is made by a ceramic layer delimited by two metallic electrodes, respectively connected to the high voltage command signal and to the ground signal of the amplifier, which is sent to the plate surface where the patches are fixed with an electro-conductive glue (Loctite 3888).

This kind of piezoelectric patch has an in-plane deformation when subject to the high voltage; the goal of active control is therefore to obtain a deformation of the piezoelectric actuators that works exactly in opposition to the one produced by the vibrations of the plate surface.
For this reason, the position of the PZT patches has been optimised by using numerical calculation of the deformation energy of the plate for its first natural frequencies, performing calculations with a self-developed computer program based on Rayleigh-Ritz method [3]. Such preliminary simulations have been realised only considering the case of empty tank and nonequipped plate.

\section{Experimental modal analyses}

Some preliminary modal analyses are carried out on the system by applying random excitation to the plate and measuring its response on a grid of eight by six points, in order to identify natural frequencies, damping ratios and modal shapes.

In particular, three complete modal analyses are performed on the plate: these are for empty tank, tank filled to half-plate and tank full of water (to $410 \mathrm{~mm}$ from the bottom).

Additional measurements are carried out (only in the excitation point) in presence of other levels of fluid in the container, in order to evaluate the influence of the progressive variation of the wetted surface of the plate on the values of the natural frequencies of the system.

All tests are repeated before and after applying the PZT patches and the accelerometers on the plate surface, in order to evaluate the influence of their presence on the system dynamics.

An electrodynamical shaker LDS V406 (driven by a LDS PA100E power amplifier) excites the plate through a thin stinger made of a stainless-steel wire, which is located between the load cell and the shaker; the stinger is connected through two dowels in order to transmit the excitation force, as shown in Fig. 2. The stinger should be as much as possible orthogonal to the plate surface, in order to avoid the transmission of other force components to the system. The excitation point has been chosen in an eccentric position on the plate surface, in order to excite a lot of modes.

The system response is measured by using a sub-miniature accelerometer (model Endevco 22, weight $0.14 \mathrm{~g}$, with sensitivity equal to $0.53 \mathrm{pC} / \mathrm{g}$ ), in order to minimise the presence of additional masses. The accelerometer is progressively moved on different points on the measurement grid, in order to reconstruct the modal shapes of the plate.

The time responses of the accelerometer and of the load cell are measured by using a Difa Scadas II front-end, connected to a HP c3000 workstation, and the software CADA-X of LMS for signal processing and data analysis. The same front-end is used to generate the excitation signal that drives the electrodynamical shaker.

The frequency response functions are estimated using the $\mathrm{H}_{\mathrm{V}}$ technique, after realising twelve averages on the measured time signals. A good coherence of the measurement is in general observed. Natural frequencies and damping ratios are estimated by using the Frequency domain Direct Parameter Identification (FDPI) technique of LMS. The model is then validated through the Modal Assurance Criterion (MAC) and the Modal Phase Collinearity (MPC). 


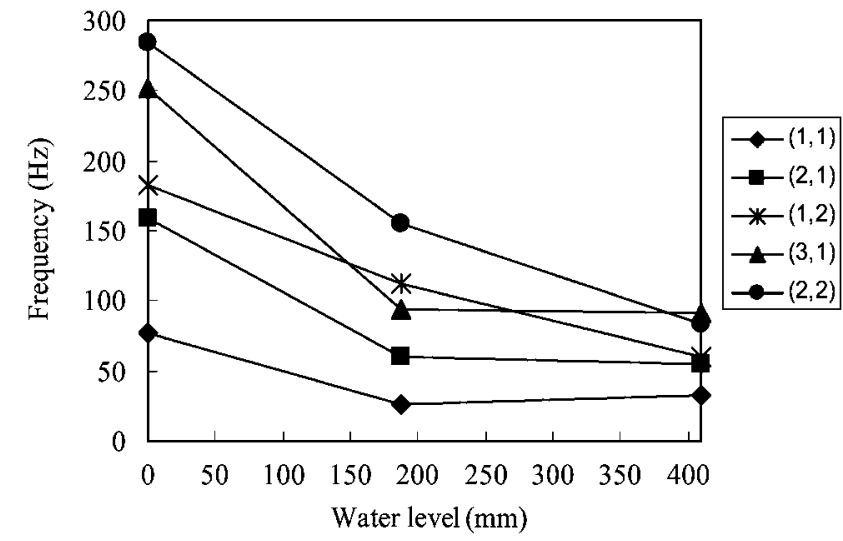

Fig. 3. Variation of the natural frequencies of the equipped plate from empty tank to half-filled tank and completely water-filled tank, for the first vibration modes.

In this paper, the identified modal shapes are referred to with the notation $(m, n)$, where $m$ and $n$ represent respectively the number of half-waves along the long and the short edge of the plate.

The first three complete modal analyses show that the progressive increment of the water level produces a progressive decrease of the natural frequencies, as shown in Fig. 3. Other experimental tests, which are realised in presence of a higher number of levels of fluid in the tank, confirm such trend.

Table 1 shows the experimentally identified modal parameters for both empty and completely water-filled tank. It should be anyway observed that the values of the natural frequencies can change significantly during the year because of variations of the environment conditions (atmospheric pressure and temperature).

Fig. 4 shows the first four modal shapes in the case of completely water-filled tank.

All tests are repeated before and after applying the PZT patches and the accelerometers on the plate surface, in order to evaluate the influence of their presence on the system dynamics. A decrease of all the natural frequencies due to the additional masses of the accelerometers and of the piezoactuators is observed in the case of empty tank. The sequence of the modal shapes is instead not significantly influenced by the introduction of the control components.

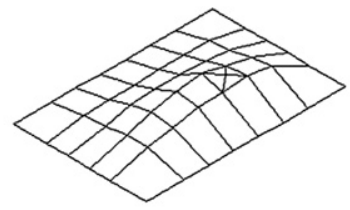

(a)

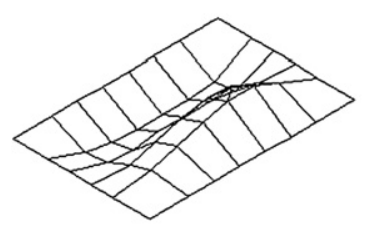

(c)

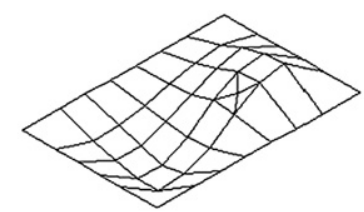

(b)

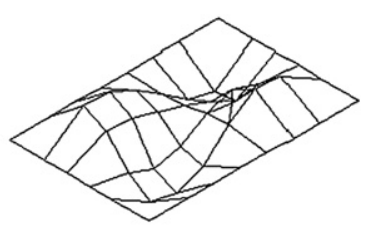

(d)
Fig. 4. First experimentally identified modal shapes, in case of completely water-filled tank and in presence of the control components: (a) mode $(1,1)$ at $33 \mathrm{~Hz}$; (b) mode $(2,1)$ at $54 \mathrm{~Hz}$; (c) mode $(1,2)$ at $60 \mathrm{~Hz}$; (d) mode $(2,2)$ at $84 \mathrm{~Hz}$. The excitation point is marked with a cross.

\section{The control system: Hardware and algorithm}

After the preliminary experimental modal analyses (realised at University of Parma, Italy), active control is experimentally applied on the plate in linear field in presence of sinusoidal disturbance (on the first three resonance peaks), testing filtered-x Least Mean Square (FXLMS) adaptive feedforward control for both empty and completely water-filled tank. A complete hardware control system has been therefore organised at ONERA/DDSS (France), as shown in Fig. 5.

FXLMS control is applied on the system with a Single Input Single Output (SISO) approach: each control channel is studied independently and the acceleration signal measured by each error sensor is influenced only by the control action of the corresponding nearly-collocated piezoelectric actuator.

In each SISO channel, the acceleration signal is firstly analogically filtered in order to avoid aliasing problems and then it is sent to a dSPACE real-time digital controller (made of one DS1104 processor Board at $250 \mathrm{MHz}$, supplied by University of Parma) passing through a 12-bit A/D converter. The controller generates the command input for the correspondent nearly-collocated PZT actuator and such signal is reconstructed by a 16-bit D/A converter. The high frequencies due to digitalisation are cut by a low-pass analogical smoothing filter. Every PZT actuator receives the command signal from a high-voltage power amplifier.

Table 1

Modal shapes, natural frequencies and damping ratios of the identified modes in case of empty tank and completely water-filled tank (in presence of the control components on the plate surface)

\begin{tabular}{|c|c|c|c|c|c|c|}
\hline \multirow{2}{*}{$\begin{array}{l}\text { Mode } \\
\text { number }\end{array}$} & \multicolumn{3}{|c|}{ Case of empty tank } & \multicolumn{3}{|c|}{ Case of completely water-filled tank } \\
\hline & $\begin{array}{l}\text { Modal } \\
\text { shape }\end{array}$ & $\begin{array}{l}\text { Frequency } \\
(\mathrm{Hz})\end{array}$ & $\begin{array}{l}\text { Damping } \\
(\%)\end{array}$ & $\begin{array}{l}\text { Modal } \\
\text { shape }\end{array}$ & $\begin{array}{l}\text { Frequency } \\
(\mathrm{Hz})\end{array}$ & $\begin{array}{l}\text { Damping } \\
(\%)\end{array}$ \\
\hline 1 & $(1,1)$ & 77.6 & 2.78 & $(1,1)$ & 32.8 & 0.67 \\
\hline 2 & $(2,1)$ & 158.7 & 2.66 & $(2,1)$ & 54.3 & 1.17 \\
\hline 3 & $(1,2)$ & 183.1 & 3.35 & $(1,2)$ & 59.9 & 1.72 \\
\hline 4 & $(3,1)$ & 251.8 & 2.27 & $(2,2)$ & 83.7 & 0.64 \\
\hline 5 & $(2,2)$ & 284.2 & 1.95 & $(3,1)$ & 91.3 & 0.61 \\
\hline
\end{tabular}




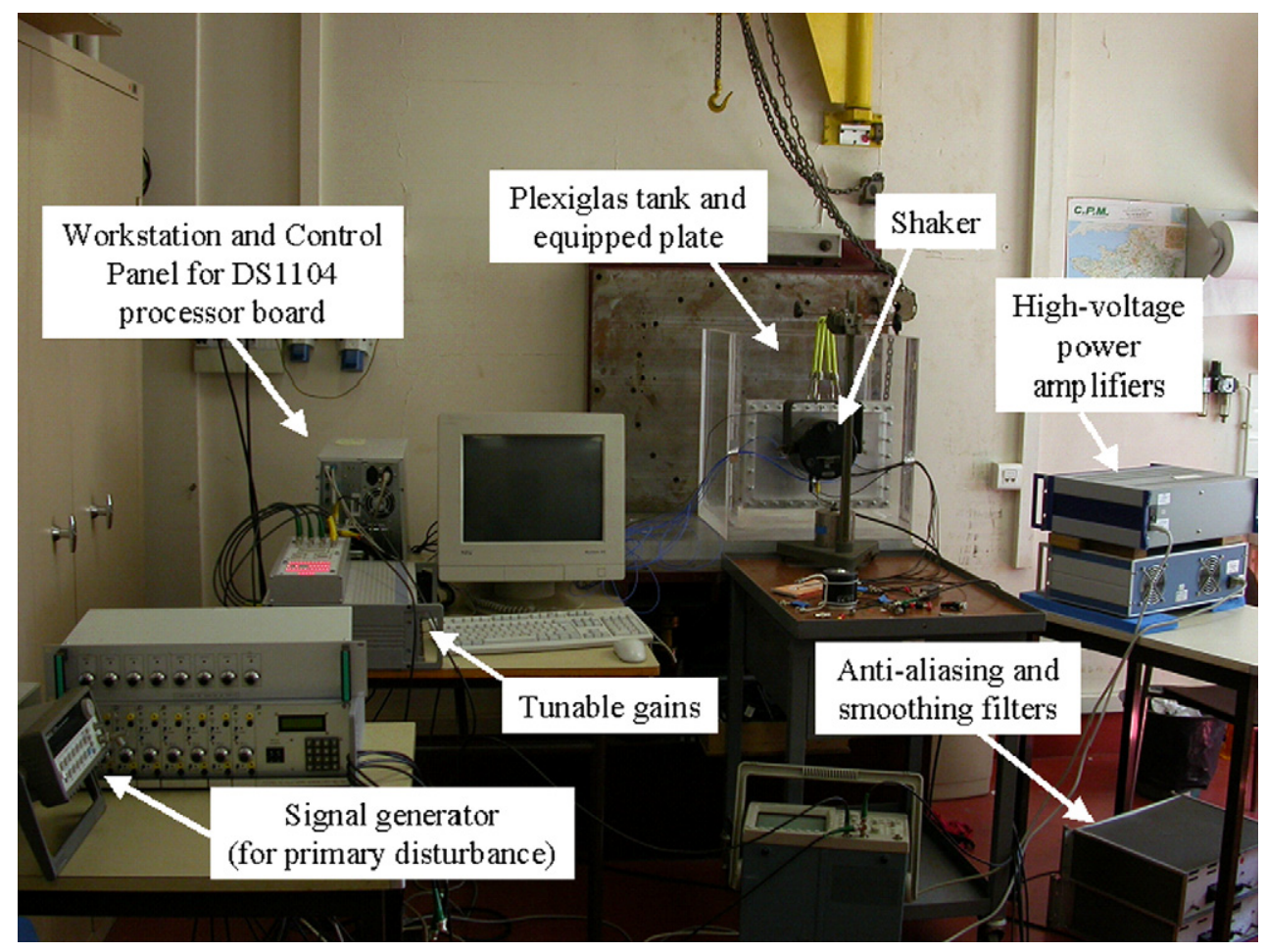

Fig. 5. The complete hardware control system.

During the control tests, the primary disturbance is produced by an electrodynamical B\&K shaker model 4809 . The load cell, which is glued on the plate in the excitation point, measures the actual force given to the plate by the shaker and this signal is used as reference signal, as required in feedforward control [5,9].

Some amplification gains are also opportunely introduced into the control-loop in order to increase the ratio signal/noise.

The control algorithm is implemented on the processor board working in a Matlab-Simulink environment and choosing a sampling frequency equal to $1000 \mathrm{~Hz}$.

The FXLMS algorithm is a classical feedforward control technique which belongs to the class of adaptive algorithms $[5,9]$. The concept of feedforward control is to derive a reference signal from the primary excitation and to use it directly to drive the control secondary input. Theoretically, in absence of noise, this technique could bring to a perfect cancellation of the primary disturbance. In real cases, the continuous changes in the excitation signal and in the primary path make necessary that the frequency response of the controller is adjusted with time, using an adaptive technique.

The model of a practical implementation of a single-channel feedforward control with FXLMS adaptive algorithm is showed in Fig. 6, where $x$ is the primary excitation (assumed as reference signal), $d$ is the response of the mechanical system in absence of control, $u$ is the secondary control input and $e$ is the error signal (that is the measured acceleration signal). A good correlation between $x$ and $d$ is required in order to obtain a significant control effectiveness [9].

The controller is represented by $H$, that is a Finite Impulse Response (FIR) filter that acts on the primary disturbance $x$ in

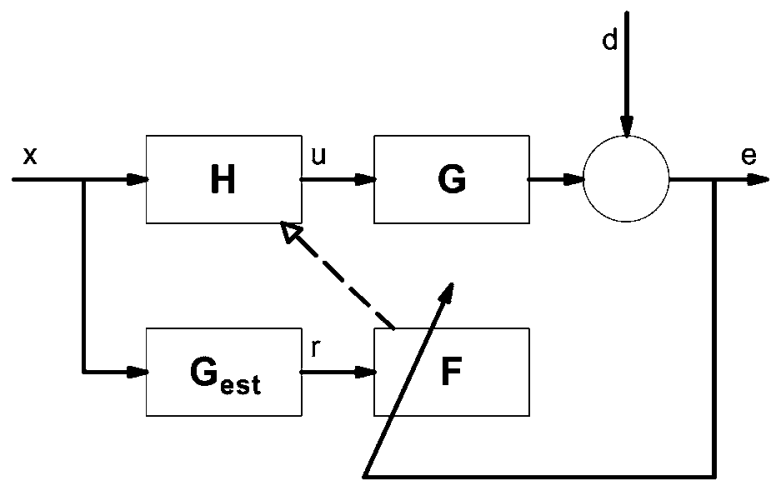

Fig. 6. Block diagram of the filtered-x LMS algorithm.

order to generate the control secondary input $u$. The coefficients of filter $H$ must be continuously changed with time, in order to have optimal control performances. For this reason, the cost function $J$ must be minimised at every sample time $n$ :

$J=e^{2}(n)$.

Such minimisation is realised by the LMS adaptive filter $F$, which calculates the coefficients $w_{i}$ of the $H$ filter

$w_{i}(n+1)=w_{i}(n)-\mu \frac{\partial J}{\partial w_{i}(n)}$

where $w_{i}(n)$ represents the $i$ th controller coefficient at the $n$th sample time and $\mu$ is a convergence parameter. Following [7], Eq. (2) can be rewritten as

$w_{i}(n+1)=w_{i}(n)-2 \mu e(n) r(n-i)$.

In order to obtain the so-called filtered reference signal $r$, the primary disturbance $x$ must be filtered with an appropriate fil- 
Table 2

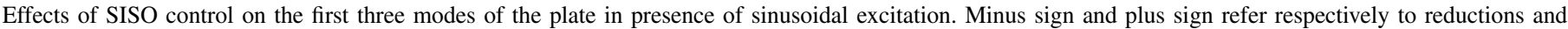
amplifications of the FFT of the error signal, expressed in $\mathrm{dB}$, at the frequencies of interest

\begin{tabular}{|c|c|c|c|c|c|c|}
\hline \multirow[t]{2}{*}{ Channel } & \multicolumn{3}{|c|}{ Control effectiveness in case of empty tank $(\mathrm{dB})$} & \multicolumn{3}{|c|}{ Control effectiveness in case of completely water-filled tank (dB) } \\
\hline & $\begin{array}{l}\text { Mode } \\
(1,1)\end{array}$ & $\begin{array}{l}\text { Mode } \\
(2,1)\end{array}$ & $\begin{array}{l}\text { Mode } \\
(1,2)\end{array}$ & $\begin{array}{l}\text { Mode } \\
(1,1)\end{array}$ & $\begin{array}{l}\text { Mode } \\
(2,1)\end{array}$ & $\begin{array}{l}\text { Mode } \\
(1,2)\end{array}$ \\
\hline 1 & -17.8 & -27.4 & -17.1 & -5.5 & -10.4 & -3.3 \\
\hline 2 & -14.2 & -39.1 & +1.0 & -4.9 & -9.2 & -0.8 \\
\hline 3 & -38.2 & -20.6 & -18.2 & -3.5 & -3.6 & -1.8 \\
\hline 4 & -23.3 & -57.4 & +3.0 & -4.4 & -19.7 & -1.7 \\
\hline 5 & -23.3 & -29.7 & -14.2 & -4.9 & -3.7 & -1.3 \\
\hline
\end{tabular}

ter $G_{\text {est }}$, which represents an estimated model of the secondary control path $G$ (transfer function between the error sensors and the PZT patches in absence of any other external perturbation). In the present study, a preliminary off-line identification of $G_{\text {est }}$ as FIR filter is realised.

\section{Experimental results of active vibration control}

FXLMS control is applied to the studied system, for both empty and completely water-filled tank, by using a SISO approach. The force measured by the load cell is used as reference signal.

The necessary identification of a model $G_{\text {est }}$ of the secondary control path [5,9] is firstly performed for every control channel, in absence of any external perturbation.

A good compromise between convergence velocity and stability of the algorithm is reached on all the control channels by optimising the value of the convergence parameter $\mu$.

The plate is subject to sinusoidal excitation, for both empty and completely water-filled tank, at its first three resonance frequencies, which have been previously identified during the experimental modal analyses. Since the natural frequencies of the system can anyway vary significantly during the year because of different environment conditions (atmospheric pressure and temperatures), during each control test the tonal primary disturbance is tuned in order to match the actual value of the natural frequencies. That justifies possible differences between the applied excitation frequencies and the natural frequencies shown in Table 1.

Table 2 shows the control effectiveness that is obtained on the control channels in presence of tonal excitation of the first three vibration modes. The reported values refer to reductions (minus sign) or amplifications (plus sign) of the Fast Fourier Transform (FFT) of the acceleration signals, expressed in $\mathrm{dB}$, at the frequencies of interest.

At first, active control is applied to the system in the case of absence of water in the Plexiglas tank. In the case of excitation of mode $(1,1)$, at about $85 \mathrm{~Hz}$, the SISO approach has a global satisfactory effect on all the control channels, with a particularly important reduction of $38 \mathrm{~dB}$ on Channel 3 (central patch); this result is confirmed by the significant reduction of the FFT of the acceleration signal at $85 \mathrm{~Hz}$ shown in Fig. 7.

Each piezoactuator is in fact located on an anti-node (position of maximum oscillation) of one of the first three modal shapes shown in Fig. 4, so it is expected to optimally control

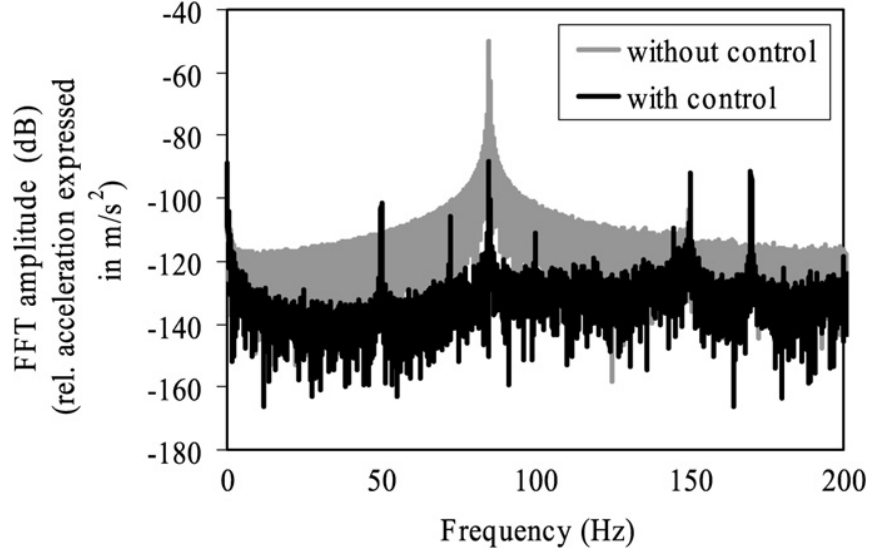

Fig. 7. Control effectiveness on the Fast Fourier Transform (FFT) of the acceleration signal on Channel 3 for the first mode at $85 \mathrm{~Hz}$ (case of empty tank).

it. In particular, Channel 3 has the theoretical best position to control the modal shape $(1,1)$.

The presence of a harmonic component at a frequency three times the fundamental one (i.e. $255 \mathrm{~Hz}$ ) is also observed: this phenomenon is typical in plates, which present hardening type nonlinearity. Its magnitude is anyway small if compared with the main component at $85 \mathrm{~Hz}$, so the system is here studied as linear and only the control of the first harmonic component is investigated.

Also the other channels show satisfactory results in controlling the first mode, even if the effectiveness of symmetrical actuators (with respect to the symmetry axes of the plate) is not exactly the same. This is probably due to a small asymmetry of the plate, produced mainly by the additional mass of the load cell, which is in eccentric position, and by possible geometrical imperfections of the plate surface.

Table 2 also shows the satisfactory results obtained especially on Channels 2 and 4 (up to $57 \mathrm{~dB}$ of reduction) in controlling the second resonant peak; in Fig. 8, the good convergence velocity of the algorithm on Channel 2 can be also observed. As explained before, Channels 2 and 4 are in fact expected to produce the best performances in controlling mode $(2,1)$ at $163 \mathrm{~Hz}$, since they are located on its anti-nodes; mode $(2,1)$ is in fact characterised by a modal shape with two half-waves along the long edge of the plate.

For the same reason, the third mode, with modal shape $(1,2)$ at $191 \mathrm{~Hz}$, is optimally controlled by actuators 1 and 5, which have the best theoretical position to control its modal shape. 


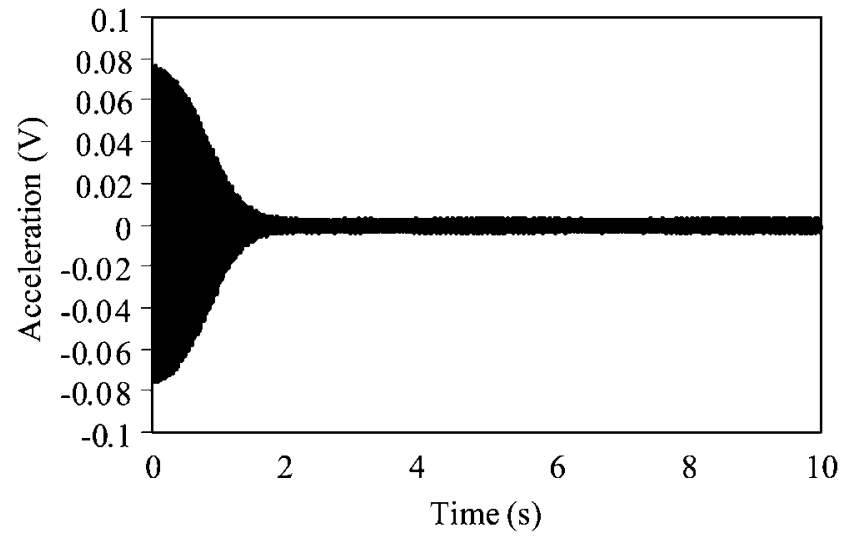

Fig. 8. Transient acceleration signal on Channel 2 during convergence of FXLMS control on the second mode at $163 \mathrm{~Hz}$ (case of empty tank).

On Channels 2 and 4 the control action is instead very difficult and it brings to a little amplification of the acceleration signal. Satisfactory reductions are anyway unexpectedly obtained also on Channel 3 (central patch).

Active control is then applied to the studied system in the case of completely water-filled tank, in order to verify the effects of the presence of the fluid on the control effectiveness.

It is assumed that gravity and sloshing effects, as well as compressibility of the fluid, do not give significant effects on the structural modes. Sloshing modes of the free surface and structural modes arise in fact in very different frequency ranges. The sloshing resonances $f_{m n}$ for a rigid rectangular container (only slightly changed by a flexible wall) are given by $[1,8]$ :

$f_{m n}=\sqrt{g k \tanh (k h)} /(2 \pi)$,

where $k$ can be calculated as

$k^{2}=\pi^{2}\left(\frac{m^{2}}{a^{2}}+\frac{n^{2}}{b^{2}}\right)$,

$g$ is the gravity acceleration, $h$ is the liquid level, $a$ and $b$ are the dimensions of the base of the tank and $m$ and $n$ are integers indicating the number of half-waves on the free surface in the two directions.

In the case of the studied system, twenty-two sloshing modes are calculated, covering globally a range from 1.3 to $3.4 \mathrm{~Hz}$. The sloshing frequencies are therefore at much lower frequencies than the first structural mode to be controlled (about $23 \mathrm{~Hz}$ in the case of completely water-filled tank) and their presence can therefore be neglected in this study.

Table 2 also shows the results that are obtained for the first three resonant peaks (respectively at about 23, 51 and $60 \mathrm{~Hz}$ ) in case of completely water-filled tank.

On first and third resonance, the reduction values (up to about $5.5 \mathrm{~dB}$ on the first mode) are smaller than in the case of empty tank and this low control effectiveness is uniform on the plate surface, since the control channels seem to loose their capability to control selectively some modal shapes.

Higher reductions (up to about $20 \mathrm{~dB}$ ) are obtained on some control channels on the second resonance peak, but it can be anyway observed that Channel 2 has lost its theoretical optimal control effectiveness on the modal shape $(2,1)$.

\section{Conclusions}

Active vibration control of a rectangular plate representing a flexible wall of a thick Plexiglas container is experimentally investigated in linear field, for both empty and water-filled tank, in presence of sinusoidal disturbance on the first three resonance peaks. Five piezoelectric PZT patches are used as control actuators and a feedforward control technique, based on a filtered-x least mean square (FXLMS) algorithm, is tested.

Before applying control, some preliminary experimental modal analyses are carried out on the system, in case of empty tank and in presence of different water levels, in order to identify natural frequencies, damping ratios and modal shapes of the plate. It is observed that the introduction of the fluid produces a significant reduction of the natural frequencies, but not very significant changes in the modal shapes. Measures are repeated before and after introducing the control components on the plate surface, obtaining the same sequence of modes.

Active control is then applied to the studied system, starting from the case of empty tank, obtaining satisfactory reductions of the error signal (up to about $57 \mathrm{~dB}$ on Channel 4 at the second resonance). In all control tests, a particular effectiveness of the theoretically optimally located actuators on the correspondent modal shapes appears.

Introducing water, the control action produces lower effects (up to about $20 \mathrm{~dB}$ on the second mode, but not more than $5.5 \mathrm{~dB}$ on first and third one) and the control channels seem to loose their capability to control selectively some modal shapes. This may be due to the small changes in the modal shapes produced by the presence of the fluid, which can limit the controllability of the system, and to higher forces required to the small PZT patches for controlling vibrations in presence of the increased modal mass of the system at lower frequency.

For these reasons, the use of more powerful piezoelectric patches could be advisable in future. Further investigations will be conducted starting from the obtained results in order to verify this hypothesis. A finite-elements model could be also used in order to optimise the locations and the dimensions of the PZT actuators even in case of fluid-structure interaction. The FXLMS algorithm could be finally experimentally applied in MultiSISO approach in order to improve the results.

\section{Acknowledgements}

The present research is carried out within the context of an Italian-French doctoral dissertation between University of Parma and CNAM-Paris by the first author.

It has been partially supported by the Italian Ministry for Research (MIUR) through the FIRB 2001 and COFIN 2003 grants and also by the Marie Curie European Doctorate in Sound and Vibration Studies (EDSVS) through a training of the first author at ONERA/DDSS (France).

\section{References}

[1] H.N. Abramson, The dynamic behaviour of liquids in moving containers, NASA SP-106, Washington, DC, 1966. 
[2] M. Amabili, Eigenvalue problems for vibrating structures coupled with quiescent fluids with free surface, Journal of Sound and Vibration 231 (1) (2000) 79-97.

[3] M. Amabili, Nonlinear vibrations of rectangular plates with different boundary conditions: Theory and experiments, Computers and Structures 82 (31-32) (2004) 2587-2605.

[4] E.K. Dimitriadis, R. Fuller, C.A. Rogers, Piezoelectric actuators for distributed vibration excitation of thin plates, Journal of Vibration and Acoustics 113 (1991) 100-107.

[5] S.J. Elliott, Signal Processing for Active Control, Academic Press, London, 2001.

[6] O.M. Fein, L. Gaul, U. Stöbener, Vibration reduction of a fluid-loaded plate by modal control, Journal of Intelligent Material Systems and Structures 16 (6) (2005) 541-552.

[7] C.R. Fuller, S.J. Elliott, P.A. Nelson, Active Control of Vibration, Academic Press Limited, London, 1996.

[8] R.A. Ibrahim, Liquid Sloshing Dynamics. Theory and Applications, Cambridge University Press, Cambridge, 2005.
[9] S.M. Kuo, D.R. Morgan, Active Noise Control Systems: Algorithms and DSP Implementations, Wiley, New York, 1996.

[10] H.K. Lee, Y.S. Park, A near-field approach to active control of sound radiation from a fluid-loaded rectangular plate, Journal of Sound and Vibration 196 (5) (1996) 579-593.

[11] S. Li, D. Zhao, Numerical simulation of active control of structural vibration and acoustic radiation of a fluid-loaded laminated plate, Journal of Sound and Vibration 272 (1-2) (2004) 109-124.

[12] H. Morand, R. Ohayon, Fluid-Structure Interactions, Wiley, Chichester, 1995.

[13] F. Peng, A. Ng, Y. Hu, Actuator placement optimization and adaptive vibration control of plate smart structures, Journal of Intelligent Material Systems and Structures 16 (3) (2005) 263-271.

[14] B. Petitjean, I. Legrain, F. Simon, S. Pauzin, Active control experiments for acoustic radiation reduction of a sandwich panel: Feedback and feedforward investigations, Journal of Sound and Vibration 252 (1) (2002) 19-36.

[15] B. Widrow, S.D. Stearns, Adaptive Signal Processing, Prentice-Hall, New Jersey, 1985. 